\title{
El papel de la personalidad en el juego problemático y en las preferencias de géneros de videojuegos en adolescentes
}

\section{The role of personality on disordered gaming and game genre preferences in adolescence: Gender differences and person-environment transactions}

\author{
Francisco J. López-Fernández*, Laura Mezquita****, Mark D. Griffiths****, Generós Ortet*,**, \\ MANUEL I. IBÁÑEZ***. \\ * Departamento de Psicología Básica, Clínica y Psicobiologia, Universitat Jaume I, Castellón, España. \\ ** Instituto de Salud Carlos III, Centro de Investigación Biomédica en Red de Salud Mental (CIBERSAM), Castellón, España. \\ *** Unidad Internacional de Estudios sobre Videojuegos, Departamento de Psicología, Nottingham Trent University, \\ Nottingham, Inglaterra, Reino Unido.
}

\section{Resumen}

Jugar a videojuegos es una de las actividades de ocio más populares en adolescentes. El principal objetivo de este estudio fue examinar los efectos aditivos y de moderación del género y la personalidad en el juego problemático y en la preferencia en géneros de videojuegos durante la adolescencia. 776 estudiantes españoles (media de edad = 14,$29 ; D T=1,59 ; 50,64 \%$ chicas) cumplimentaron cuestionarios del Modelo de los cinco grandes de personalidad y de conductas relacionadas con videojuegos. Se observaron diferencias de género en conductas relacionadas con videojuegos: los chicos jugaban más y presentaron mucho más uso problemático que las chicas. Ellos prefirieron géneros competitivos; por ejemplo, juegos de acción-shooters, deportes, lucha y estrategia. Las chicas prefirieron géneros no violentos y ocasionales; por ejemplo, simulación social, y juegos de habilidad y lógica. El género moderó las asociaciones entre personalidad y juego problemático: el juego problemático se asoció a baja amabilidad y baja responsabilidad en chicos, y a baja extraversión y baja responsabilidad en chicas. La baja responsabilidad moderó las asociaciones entre frecuencia de juego y uso problemático: jugar más a videojuegos conducía a un uso problemático de éstos, principalmente en individuos irresponsables e impulsivos. Se encontraron asociaciones pequeñas pero significativas entre la personalidad y preferencias en géneros de videojuegos. Estos hallazgos destacan la relevancia del género y la personalidad en las conductas relacionadas con videojuegos durante la adolescencia, y animan a prestar más atención a las diferencias dependientes del género y a las transacciones persona-ambiente al estudiar estas conductas.

Palabras clave: Videojuegos; adicción; géneros de videojuegos; personalidad; género.

\begin{abstract}
Playing video games is one of the world's most popular leisure activities, especially for teenagers. The main aim of the present study was to examine additive and moderation effects of gender and personality to explain individual differences in problematic gaming and video game genre preferences in adolescence. 776 Spanish high school students (mean age $=14.29$ years, $S D=1.59,50.64 \%$ girls) completed the questionnaires of the Five-Factor Model of personality, frequency of video gaming, disordered use, and the video games they mostly played. Gender differences were observed for gaming behaviors: boys played more and presented much more disordered gaming than girls. Boys preferred competitive genres; for example, action-shooters, sport, fight and strategy games. Girls preferred nonviolent and ocasional game genres; for example, social simulation, and brain and skill games. Gender moderated the association between personality and disordered gaming: disordered gaming was associated with low agreeableness and low conscientiousness in boys, and with low extraversion and low conscientiousness in girls. Low consciousnness moderated the association between gaming frequency and problematic use of video games: playing more video games led to disordered gaming, mainly in irresponsible and impulsive individuals. Though small, significant associations were found among all of the personality domains and vid- eo game genre preferences. These findings highlight the relevance of gender and personality for gaming behaviors in adolescence, and suggest paying more attention to gender-dependent differences and person-environment transactional processes when studying gaming-related behaviors.

Keywords: Video games; addiction; game genres; personality; gender.
\end{abstract}

Recibido: Julio 2019; Aceptado: Octubre 2019.

Enviar correspondencia a: Manuel I. Ibáñez.

Dpto. de Psicología Básica, Clínica y Psicobiologia. Universitat Jaume I. Av. de Vicent Sos Baynat, s/n, 12071 Castellón, España. Tel. +34 964729690 E-mail: iribes@uji.es 
$\mathrm{E}$ n las últimas décadas, jugar a videojuegos se ha convertido en una de las actividades de ocio más populares entre los adolescentes a nivel mundial (Entertainment Software Association -ESA-, 2018). Aproximadamente el $47 \%$ de la población española juega a videojuegos regularmente (Asociación Española de Videojuegos-AEVI-, 2018). Para una minoría de jugadores, el juego puede desencadenar efectos negativos similares a componentes adictivos de otros trastornos relacionados con adicciones, incluyendo saliencia, cambios de humor, tolerancia, abstinencia o recaída (Griffiths, 2005). El DSM5 (American Psychiatric Association -APA-, 2013) ha incluido el Trastorno de juego por internet (IGD, según sus siglas en inglés) como una potencial adicción conductual merecedora de más investigación. Además, recientemente, la adicción a los videojuegos ha sido reconocida en el ICD-11 (Bobes, Flórez, Seijo y Bobes, 2019; Organización Mundial de la Salud-OMS-, 2017).

La prevalencia del juego problemático se encuentra entre el 1-9\%, dependiendo de criterios de corte, edad, sexo o diferencias socioculturales (Gentile, 2009; Gentile et al., 2017; Mihara y Higuchi, 2017; Paulus, Ohmann, Von Gontard y Popow, 2018). El juego problemático puede generar aislamiento y bajo rendimiento académico (Gentile et al., 2011; Lemmens, Valkenburg y Peter, 2011), y ha mostrado asociaciones altas con síntomas de trastornos afectivos, emocionales, y por déficit de atención e hiperactividad (González-Bueso et al., 2018; Mihara y Higuchi, 2017; Müller et al., 2015). Por tanto, una mayor comprensión de los factores facilitadores del juego problemático ayudaría a prevenir tales efectos perjudiciales.

La personalidad es un factor de riesgo para el IGD, y por ello ha sido evaluada en muchos estudios (Gervasi et al., 2017; Mihara y Higuchi, 2017; Paulus et al., 2018; Șalvarlı y Griffiths, 2019). Los rasgos de personalidad son relevantes para una gran variedad de aspectos vitales (Ozer y Benet-Martínez, 2006), incluyendo diversas conductas relacionadas con las adicciones (Andreassen et al., 2013; Ibáñez et al., 2010; Mezquita et al., 2015). Hoy en día, el marco de personalidad más aceptado es el Modelo de los cinco grandes (FFM) (John, Naumann y Soto, 2008). Éste propone cinco dimensiones básicas: extraversión, neuroticismo, amabilidad, responsabilidad, y apertura a la experiencia (McCrae y Costa, 2008). De acuerdo a revisiones sistemáticas recientes, las áreas de personalidad del FFM que más consistentemente se relacionan con el IGD son neuroticismo, baja responsabilidad, y baja amabilidad (Gervasi et al., 2017; Șalvarlı y Griffiths, 2019).

La mayoría de estos estudios se ha realizado en población adulta (ej., Braun, Stopfer, Müller, Beutel y Egloff, 2016; Charlton y Danforth, 2007). Por tanto, no se ha contrastado su relevancia para la preadolescencia y adolescencia. Esto supone un gran vacío en la investigación, ya que esta etapa vital es de especial importancia en cuanto al de- sarrollo de problemas psicológicos asociados al juego problemático de videojuegos (Mihara y Higuchi, 2017; Paulus et al., 2018). Muy pocos estudios han explorado la relación entre las dimensiones del FFM y el juego problemático en preadolescentes y adolescentes. En estas edades, el juego problemático se ha asociado, de forma consistente, con baja responsabilidad (García-Oliva y Piqueras, 2016; Vollmer, Randler, Horzum y Ayas, 2014; Wang, Ho, Chan y Tse, 2015). No obstante, los resultados son menos concluyentes en relación a otras áreas del FFM: dos estudios lo han asociado con baja extraversión (García-Oliva y Piqueras, 2016; Vollmer et al., 2014), mientras que solo un estudio lo ha asociado con baja amabilidad, alto neuroticismo (Vollmer et al., 2014) y baja apertura a la experiencia (Wang et al., 2015). Estos escasos y distintos hallazgos muestran la necesidad de investigar más los efectos aditivos de la personalidad sobre el juego problemático durante la adolescencia, el principal objetivo de nuestro estudio.

Otro tema que ha sido explorado escasamente en el campo de los videojuegos y la personalidad son las transacciones persona-ambiente; es decir, cómo la personalidad se interrelaciona de manera compleja con el entorno para influir en la conducta (Caspi y Roberts, 2001). Dichos procesos se han descrito en el campo de los videojuegos y la agresividad. Algunos estudios sugieren que la exposición a videojuegos violentos puede fomentar conductas agresivas, pero principalmente en personas con personalidad agresiva (ej., Anderson y Dill, 2000; Markey y Markey, 2010). Dichos hallazgos pueden indicar una transacción reactiva; es decir, personas distintas reaccionan de manera diferente ante un mismo entorno, según sus rasgos de personalidad (Caspi y Roberts, 2001). No obstante, y que sepamos, queda inexplorado el papel de las transacciones persona-ambiente en el desarrollo del juego problemático. Un factor ambiental de riesgo para el IGD es la cantidad de tiempo dedicado a jugar a videojuegos (Gentile, 2009; Mihara y Higuchi, 2017), aunque solo una minoría de jugadores regulares tiende a desarrollar juego problemático (Charlton y Danforth, 2007). Esto puede sugerir una transacción persona-ambiente. Es decir, una mayor frecuencia de juego tendría un impacto más negativo sobre algunos jugadores, y no otros, debido a sus características personales. El segundo objetivo de nuestro estudio es explorar si la personalidad modera la relación entre una mayor frecuencia de juego y el juego problemático en videojuegos.

Además, la personalidad puede ser relevante para las preferencias de géneros de videojuegos; que sepamos, este asunto no ha sido examinado en adolescentes. Algunos estudios sugieren que los adultos con baja amabilidad preferirían videojuegos violentos (Chory y Goodboy, 2011; Greitemeyer y Sagioglou, 2017), jugadores extrovertidos preferirían juegos de acción, y jugadores con alta apertura a la experiencia optarían por juegos de rol (RPG) (Braun et al., 2016). También se ha sugerido que algunos géneros 
pueden ser potencialmente más adictivos que otros (Rehbein, Staudt, Hanslmaier y Kliem, 2016). En particular, se ha demostrado que la preferencia por juegos de RPG, shooter y simulación contribuyen a más tiempo de juego (Rehbein et al., 2016), y que los juegos RPG, especialmente los MMORPG (Massively Multiplayer Online Role-Playing Games), y shooter, tienden a una mayor asociación con el juego problemático (Lemmens y Hendrix, 2016; Müller et al., 2015). Por tanto, explorar las características de personalidad relacionadas con las preferencias de géneros de videojuegos en la adolescencia, el tercer objetivo de nuestro estudio, también es una cuestión relevante.

Por último, el género también es otro factor relevante en las conductas relacionadas con el juego. Los chicos juegan más a videojuegos y tienen mucho más juego problemático que las chicas (Mihara y Higuchi, 2017; Paulus et al., 2018). Ellos también tienden a preferir géneros de videojuegos más competitivos y agresivos; por ejemplo, acción-shooters o juegos deportivos. Las chicas tienden a preferir juegos más ocasionales y no violentos; por ejemplo, puzles o videojuegos de plataforma (Lemmens y Hendriks, 2016; Rehbein et al., 2016; Scharkow, Festl, Vogelgesang y Quandt, 2015). Aún así, a pesar de estas diferencias claras de género, apenas se ha examinado la posibilidad de que los factores de riesgo para el juego problemático difieran entre chicos y chicas. En cuanto a la personalidad, únicamente un estudio, que sepamos, ha explorado la asociación entre personalidad y juego problemático en chicos y chicas adolescentes por separado (Garcia-Oliva y Piqueras, 2016). Encontró que la baja responsabilidad y la baja extraversión se asociaban con adicción a los videojuegos en chicos, pero no halló efectos significativos de la personalidad para chicas. Aunque estos hallazgos preliminares se han de replicar, indican que merece atención el papel potencial de la personalidad según el género en la conducta relacionada con los videojuegos, nuestro cuarto objetivo.

En resumen, revisamos algunos vacíos importantes respecto al papel del género y la personalidad en las conductas relacionadas con videojuegos durante la adolescencia. Nuestro objetivo principal es realizar una exploración sistemática de dichas lagunas. En concreto, examinaremos el papel aditivo del género y las características de personalidad del FFM en el juego problemático y en las preferencias de géneros de videojuegos. Además, exploraremos el papel moderador (a) de la personalidad en la relación entre frecuencia de juego y juego problemático; y (b) del género en la relación entre la personalidad y las conductas relacionadas con videojuegos. Nuestras hipótesis son que los chicos presentarán más juego problemático que las chicas, y que ellos preferirán géneros competitivos y agresivos, mientras que ellas optarán por juegos más ocasionales y de puzles. Respecto a la personalidad, la baja responsabilidad y la baja extraversión se asociarán con juego problemático, principalmente entre los chicos. Debido a la escasez de es- tudios sobre la personalidad y las preferencias de género, no proponemos hipótesis sistemáticas. No obstante, es de esperar que la baja amabilidad se asocie a géneros competitivos, la extraversión a juegos de acción, y la apertura a la experiencia a los RPGs. Por último, según hallazgos previos sobre otros temas, como videojuegos violentos y conducta agresiva, planteamos la hipótesis de que habrá una interacción entre dichos factores de riesgo de la personalidad para juego problemático con la frecuencia de juego que se relacione a juego problemático.

\section{Método}

\section{Participantes y procedimiento}

Reclutamos a los participantes de dos institutos públicos en la zona metropolitana de Castellón de la Plana, en el este de España. De los 1106 estudiantes invitados a participar, 835 entregaron el consentimiento parental firmado. De éstos, 59 participantes no acudieron a las sesiones de valoración o no completaron todos los cuestionarios. La muestra definitiva está compuesta de 776 adolescentes (393 chicas), con edades entre 12-17 años, y edad media de 14,29 años (DT = 1,59).

Este estudio transversal y ex post facto (Montero y León, 2005) formó parte de una investigación más amplia sobre factores de riesgo psicosociales involucrados en la salud mental durante la adolescencia. Los asistentes de investigación administraron una batería de cuestionarios en tres sesiones de una hora, separadas por una semana. Los estudiantes cuyos padres/madres/tutores legales autorizaron su participación completaron voluntariamente una encuesta de datos sociodemográficos y la batería de cuestionarios autoadministrados en formato de papel y lápiz. Los asistentes de investigación dieron instrucciones detalladas a los estudiantes, hicieron hincapié en la confidencialidad de los datos y la importancia de la veracidad de sus respuestas, y ayudaron a los estudiantes en su caso (para más detalles referirse a Moya-Higueras et al., 2020).

\section{Medidas}

Usamos el JS NEO-A60 (Walker, López y Mezquita, 2018) para evaluar las dimensiones de la personalidad del FFM: neuroticismo, extraversión, apertura a la experiencia, amabilidad y responsabilidad. Esta escala es la versión abreviada de 60 ítems de la versión Junior Spanish del NEO-PI-R (Costa y McCrae, 1992), específicamente el JS NEO (Ortet y cols., 2012). Dicho instrumento replicó satisfactoriamente la estructura factorial del NEO-PI-R para adultos en muestras con edades entre 12-17 años y mostró puntuaciones adecuadas de fiabilidad (cada rasgo de personalidad mostró un coeficiente $\alpha>$,82) y validez de constructo (aportamos un análisis factorial conjunto del test y del cuestionario FFM enfocado a niños). Los Alfas de Cronbach para este estudio fueron ,83 para neuroticismo, 
,83 para extraversión, ,75 para apertura a la experiencia, ,82 para amabilidad y ,89 para responsabilidad.

Reportamos la frecuencia de juego en una escala tipo Likert de 6 puntos (de $0=$ "nunca o casi nunca" a 5 = "entre 3 y 5 horas diarias"). Además, los jugadores informaron de hasta cinco videojuegos más a menudo jugados, clasificados según estudios previos (ej., Lemmens y Hendriks, 2016; Rehbein et al., 2016) como: acción-shooter (ej., Call of Duty); deportes (ej., FIFA, también juegos de conducción como MotoGP); estrategia (principalmente juegos MOBA, ej., Clash Royale); lógica+habilidad (incluyendo géneros altamente intercorrelacionados (Rehbein et al., 2016): juegos de lógica de puzles, ej., Candy Crush Saga; juegos de habilidad de puzles, ej., Piano Tiles; juegos de ejercicios, ej., Wii Sports; juegos de destreza de plataforma, ej., Super Mario Bros); aventura (incluyendo juegos de aventura sin componentes shooter, ej., Assassins' Creed); simulación social (ej., The Sims); construcción (ej., Minecraft); RPG (ej., Skyrim, incluyendo MMORPG, ej., World of Warcraft); y lucha (ej., Mortal Kombat).

Usamos la adaptación española de una escala de juego problemático para jóvenes (Gentile, 2009). Realizamos la traducción inversa de la escala original de 11 ítems, incluyendo componentes adictivos, como saliencia, cambios de humor, tolerancia, abstinencia o recaída. Los participantes considerados jugadores patológicos según el estudio de la escala original dedicaban más tiempo al juego y tenían peor rendimiento académico y problemas de atención (Gentile, 2009). En este estudio, los participantes indicaron, en una escala tipo Likert de 4 puntos (de 0 = "nunca o casi nunca" a 3 "casi siempre o siempre"), la frecuencia con la cual habían tenido problemas relacionados con los videojuegos en los últimos 12 meses. Según el análisis paralelo implementado con Monte Carlo PCA (Watkins, 2006), obtuvimos una estructura unifactorial, en la cual todos los ítems presentaron cargas factoriales adecuadas, entre ,49 y ,81. El Alfa de Cronbach de esta muestra fue de ,88. Aplicamos el procedimiento del estudio original (Gentile, 2009) para fijar el punto de corte para la clasificación de juego problemático. Específicamente, codificamos las categorías "casi siempre o siempre" " "muchas veces" como 1 , la categoría "a veces" como $, 5, \mathrm{y}$ "nunca o casi nunca" como 0. Los adolescentes que mostraron al menos 6 de los 11 criterios evaluados por la escala fueron considerados jugadores patológicos.

\section{Análisis estadístico}

Usamos la versión 21 del software IBM SPSS para calcular estadísticos descriptivos, correlaciones, y los análisis t y de regresión lineal múltiple. Los valores perdidos que representaban menos del $5 \%$ en el cuestionario fueron sustituidos por la puntuación media de los ítems restantes de la escala correspondiente. Usamos InterActive, un software de código abierto de análisis y visualización de datos (McCabe, Kim y King, 2018), para mostrar gráficamente las interacciones entre los factores de riesgo de la personalidad y la frecuencia de juego en el juego problemático.

\section{Ética}

El Comité de Ética de la Universitat Jaume I aprobó el estudio, y tanto las Juntas Directivas de los institutos participantes como las autoridades regionales de la Generalitat Valenciana lo autorizaron. Los participantes y sus padres/ madres tutores legales recibieron información sobre el estudio y firmaron el consentimiento informado. Todos los procedimientos del estudio se implementaron según la Declaración de Helsinki.

\section{Resultados}

En cuanto a los datos descriptivos, 560 de los 776 participantes informaron haber jugado a videojuegos durante el último mes, 92,69\% para 383 chicos y 52,16\% para 393 chicas. Además, el 38,9\% de los chicos y el 8,3\% de las chicas jugaban a diario. El 6,4\% de todos los participantes eran «jugadores problemáticos», el 11,1\% eran chicos (43) y el $0,8 \%$ eran chicas (3). Los análisis $t$ mostraron que las chicas tenían una menor frecuencia de juego ( $d$ de Cohen $=1,26$, $p<, 001)$, y puntuaciones más altas en apertura a la experiencia $(d$ de Cohen $=, 54, p<, 001)$, neuroticismo ( $d$ de Cohen $=, 43, p<, 001)$, amabilidad ( $d$ de Cohen $=, 30, p<, 001) y$ responsabilidad ( $d$ de Cohen $=, 23, p<, 001$ ) que los chicos.

Se emplearon análisis de regresión lineal múltiple para explorar si la personalidad era predictora de frecuencia de juego, pero solo la apertura a la experienca presentó una asociación significativa que explicó el $1,3 \%$ de la varianza $(b=, 08, p=, 01)$ tras controlar por edad y género (edad; $b=$ -,05, $p=, 070$; género; $b=, 54, p=, 000)$. Exploramos el papel aditivo de la edad, el género, la personalidad y la frecuencia de juego sobre el juego problemático. También, en un último paso examinamos: a) si el género moderaba las asociaciones de personalidad y la frecuencia de juego con el juego problemático; y b) el papel moderador de la personalidad en la relación entre frecuencia de juego y juego problemático. Incluimos todas las interacciones potenciales en este último paso del modelo de regresión para controlar por posibles variables de confusión, según las recomendaciones de Keller (2014). Hallamos interacciones entre personalidad y frecuencia de juego (responsabilidad $\mathrm{x}$ juego, $b=-, 09 ; p=$ ,007) y también entre género y personalidad (amabilidad $\mathrm{x}$ género, $b=, 09 ; p=, 008$; responsabilidad x género; $b=, 15$; $p=, 000)$. Estas últimas dos interacciones indicaron que la asociación entre algunas áreas de personalidad y juego problemático diferían entre chicos y chicas. Por tanto, hicimos el análisis de regresión independientemente por género.

La Tabla 1 muestra análisis de regresión para la muestra íntegra, y también para chicos y chicas por separado. La baja responsabilidad se asoció con juego problemático para ambos géneros. La baja amabilidad se relacionó con juego 
problemático para chicos, mientras que la baja extraversión y la alta apertura a la experiencia se asoció con juego problemático para chicas. La frecuencia de juego también se relacionó con juego problemático para ambos, chicos y chicas. Se halló una interacción significativa entre frecuencia de juego y baja responsabilidad para ambos géneros.

La Figura 1 es una representación gráfica de las interacciones halladas en los análisis de regresión. Muestra el impacto negativo de la frecuencia de juego sobre el juego problemático, según distintos niveles de responsabilidad como moderador. Los resultados muestran que la frecuencia de juego tuvo una alta asociación con juego problemático en niveles bajos de responsabilidad, mientras que no hubo asociaciones en niveles altos de responsabilidad.

Realizamos los análisis de regresión lineal múltiple para cada género de videojuegos con 547 participantes (375 chicos y 172 chicas) que habían nombrado al menos un videojuego. Exploramos el papel de la edad, el género y la personalidad, y de interacciones entre género-personalidad. No hallamos efectos de moderación del género sobre la mayoría de los géneros de videojuegos, con la excepción
Tabla 1. Regresiones lineales múltiples de juego problemático como variable dependiente.

\begin{tabular}{|c|c|c|c|c|}
\hline & & \multicolumn{3}{|c|}{ Juego problemático } \\
\hline & & $\begin{array}{l}\text { Muestra total } \\
(776)\end{array}$ & $\begin{array}{c}\text { Chicos } \\
\text { (383) }\end{array}$ & $\begin{array}{c}\text { Chicas } \\
\text { (393) }\end{array}$ \\
\hline \multicolumn{5}{|l|}{ Paso 1} \\
\hline & Género &,$- 44^{\star \star \star}$ & & - \\
\hline & Edad & $07^{\star}$ & 09 &, 05 \\
\hline & $\Delta R^{2}$ &, $20 \star \star \star$ & 01 & 01 \\
\hline \multicolumn{5}{|l|}{ Paso 2} \\
\hline & Neuroticismo (N) & ,04 & 09 & ,03 \\
\hline & Extraversión (E) &,- 06 &,- 01 &,$- 20^{\star \star \star}$ \\
\hline & $\begin{array}{l}\text { Apertura a la } \\
\text { experiencia (AP) }\end{array}$ & ,06 & ,04 &, $11^{*}$ \\
\hline & Amabilidad (A) &,$- 13^{\star \star}$ &,$- 17^{\star \star \star}$ &,- 05 \\
\hline & Responsabilidad (R) &,$- 18^{\star \star \star}$ &,$- 27 * \star \star$ &,$- 12^{\star}$ \\
\hline & $\Delta R^{2}$ &, $07^{\star \star \star}$ &, $15^{\star \star \star}$ &, $08^{\star \star \star}$ \\
\hline \multicolumn{5}{|l|}{ Paso 3} \\
\hline & Frecuencia de juego &, $25^{\star \star \star}$ &, $24^{\star \star \star}$ &, $30^{\star \star \star}$ \\
\hline & $\Delta R^{2}$ &, $04^{\star \star \star}$ &, $06^{\star \star \star}$ &, $09^{\star \star \star}$ \\
\hline \multicolumn{5}{|l|}{ Paso 4} \\
\hline & NxFrecuencia de juego & ,01 & ,04 & ,02 \\
\hline & ExFrecuencia de juego & ,00 & 01 &,- 03 \\
\hline & APFrecuencia de juego &, 01 &, 00 &, 05 \\
\hline & AxFrecuencia de juego &, 05 &, 05 & ,09 \\
\hline & RxFrecuencia de juego &,$- 08^{*}$ &,$- 11^{*}$ &,$- 13^{*}$ \\
\hline & $\Delta R^{2}$ & ,01 & ,01 &, 02 \\
\hline & $\mathrm{R}^{2}$ &, 32 &, 22 & ,19 \\
\hline
\end{tabular}

Nota. Los chicos fueron asignados como 1 y las chicas como 2 ${ }^{\star} p<, 05 .{ }^{* \star} p<, 01 .{ }^{* \star \star} p<, 001$.
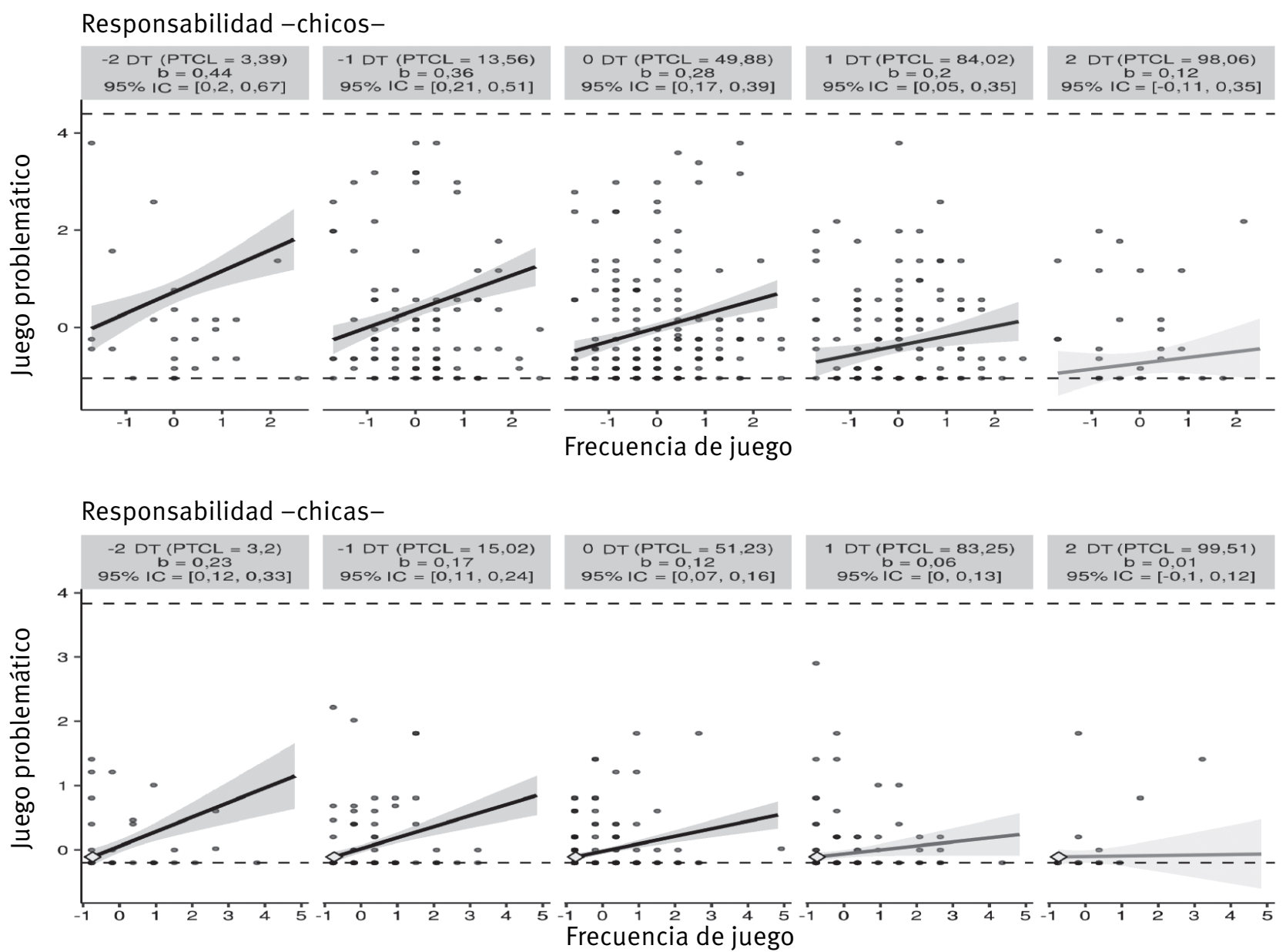

Figura 1. Gráficos de los efectos moderadores entre la responsabilidad y la frecuencia de juego sobre el juego problemático. Se muestran pendientes simples para los niveles del moderador 2 DT y 1 DT por debajo de la media, en la media, y 1 DT y 2 DT por encima de la media. Cada gráfico muestra la región calculada para el intervalo de confianza de $95 \%$ (área sombreada), los datos observados (círculos grises), los valores máximos y mínimos del resultado (líneas discontinuas horizontales), y el punto de cruce (diamante). Los ejes X representan el rango íntegro del predictor focal.

IC = intervalo de confianza; PTCL = percentil. 
de juegos de acción-shooter (extraversión x género; $b=-, 09$; $p=, 032$ ) y simulación social (apertura a la experiencia $\mathrm{x}$ género; $b=, 10 ; p=, 03)$. La Tabla 2 muestra el análisis de regresión para la muestra íntegra. En cuanto a género y preferencias en género de videojuegos, los coeficientes de la regresión indicaron que los chicos preferían juegos más competitivos (juegos de acción-shooter, deporte, estrategia y lucha) mientras que las chicas preferían juegos de simulación social y lógica+habilidad. En cuanto a personalidad, todas las dimensiones presentaron asociaciones menores con las preferencias de género. Los géneros más jugados (acción-shooter, deportes) mostraron un patrón similar de asociaciones: adolescentes con menos apertura a la experiencia y más extraversión preferían estos juegos (para acción-shooter solo detectamos este patrón para chicos; extraversión, $b=$
$, 15 ; \mathrm{p}=, 006$; apertura a la experiencia, $b=-, 10 ; p=, 069)$. Los juegos de estrategia se asociaron con baja amabilidad. La clasificación de lógica+habilidad se relacionó con apertura a la experiencia y responsabilidad. Los juegos de simulación social y aventura presentaron un patrón similar de asociaciones: los adolescentes con más apertura a la experiencia y menos extraversión preferían estos géneros (en juegos de simulación social, los efectos eran más evidentes en las chicas; extraversión, $b=-, 15 ; p=, 073$; apertura a la experiencia, $b=, 14 ; p=, 085)$. Los adolescentes con mayor neuroticismo preferían los juegos de construcción. Los juegos de lucha se asociaron con baja amabilidad, baja responsabilidad y apertura a la experiencia. Finalmente, la apertura a la experiencia, baja responsabilidad e introversión fueron predictoras de la preferencia por los RPGs.

Tabla 2. Regresiones lineales múltiples de género de videojuego como variable dependiente.

\begin{tabular}{|c|c|c|c|c|c|c|c|c|c|c|}
\hline & $\begin{array}{l}\text { Género de juego } \\
\text { (n de jugadores) }\end{array}$ & $\begin{array}{l}\text { Acción } \\
\text {-shooter } \\
\text { (292) }\end{array}$ & $\begin{array}{l}\text { Deportes } \\
\text { (251) }\end{array}$ & $\begin{array}{l}\text { Estrategia } \\
\quad(175)\end{array}$ & $\begin{array}{c}\text { Lógica } \\
+ \text { Habilidad } \\
\quad(152)\end{array}$ & $\begin{array}{l}\text { Aventura } \\
\quad(65)\end{array}$ & $\begin{array}{c}\text { Simulación } \\
\text { social } \\
\text { (47) }\end{array}$ & $\begin{array}{c}\text { Construcción } \\
\text { (40) }\end{array}$ & $\begin{array}{l}\text { Lucha } \\
\text { (32) }\end{array}$ & $\begin{array}{l}\text { RPG } \\
\text { (31) }\end{array}$ \\
\hline \multirow[t]{3}{*}{ Paso 1} & Género & 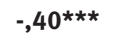 &,- 26 *** & 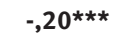 &, $41 * \star *$ &,- 06 &, $35^{\star * *}$ &,- 09 &,- 16 *** &,- 06 \\
\hline & Edad &,- 05 &,- 01 &,- 02 &,- 07 &,- 03 &,$- 12 \star *$ &,$- 14^{\star \star}$ & ,01 & ,02 \\
\hline & $\Delta \mathrm{R}^{2}$ &, $15^{\star \star \star}$ &, $06 * \star *$ &, $04^{\star \star *}$ &, $17^{\star \star \star}$ & ,01 &, $15^{\star \star \star}$ &, $03 * *$ &, $02 * \star$ &, 00 \\
\hline \multirow[t]{7}{*}{ Paso 2} & Neuroticismo &, 03 &,- 05 &,- 04 &, 03 &, 02 &,- 02 &, $17^{\star \star \star}$ &,- 08 &, 05 \\
\hline & Extraversión &, $10 *$ & ,09* &,- 05 &, 05 &,$- 09 *$ &,$- 09 *$ & 01 &, 00 &,$- 10 *$ \\
\hline & $\begin{array}{l}\text { Apertura a la } \\
\text { experiencia }\end{array}$ &,$- 11 *$ &,$- 10 \star \star$ &, 04 &, $12 * *$ &, $10 *$ &, $09 *$ & ,06 &, $10 *$ &, $19 * * *$ \\
\hline & Amabilidad &, 04 & ,03 &,$- 14^{\star \star}$ &, 05 &,- 03 &,- 03 &, 04 &,$- 16 * *$ &,- 02 \\
\hline & Responsabilidad &, 06 &,- 03 &,- 01 &, $11^{*}$ &,- 03 & ,01 &, 00 &,$- 11 *$ &,$- 11 *$ \\
\hline & $\Delta \mathrm{R}^{2}$ &, $02 *$ & $02 *$ &, 02 &, $04^{*}$ &, 02 &, 01 &, $03 * *$ &, $04^{\star \star}$ &, $05^{\star \star *}$ \\
\hline & $\mathrm{R}^{2}$ &, 17 &, 10 &, 06 &, 21 & ,01 & ,16 & ,06 &, 05 &, 05 \\
\hline
\end{tabular}

Nota. $\mathrm{RPG}=$ Juego de rol; $\mathrm{b}=$ beta normalizada; $\Delta \mathrm{R}^{2}=$ cambio en la varianza; $\mathrm{R}^{2}=\mathrm{R}^{2}$ total. Los chicos fueron asignados como 1 y las chicas como 2 .

${ }^{\star} p<, 05 .{ }^{* \star} p<, 01,{ }^{* \star *} p<, 001$.

\section{Discusión}

Este estudio muestra la relevancia de la personalidad y el género en el juego problemático y en la preferencia de géneros de videojuegos entre los adolescentes. En línea con nuestras hipótesis, los chicos mostraron mucho más juego problemático que las chicas, y preferían otros tipos de género de videojuegos. Además, perfiles específicos de personalidad influían modestamente en la preferencia por determinados géneros de videojuegos. La personalidad también mostró un papel relevante sobre el juego problemático; la baja responsabilidad para ambos géneros -chicos y chicas- y otras dimensiones del FFM mostraron asociaciones dependientes del género; la baja amabilidad en chicos, e introversión y alta apertura en chicas. Por último, también hallamos una transacción persona-ambiente en el juego problemático: una mayor frecuencia de juego impactaba más negativamente a algunos jugadores que a otros, en parte debido a sus rasgos de personalidad. Así, los adolescentes con baja responsabilidad que jugaban a videojuegos con más frecuencia tendían a presentar niveles mayores de juego problemático, en comparación a otros adolescentes que jugaban también habitualmente.

El 72,16\% de nuestra muestra había jugado a videojuegos en el último mes, un porcentaje muy similar al reportado por jugadores jóvenes en España (Buiza-Aguado, Alonso-Canovas, Conde-Mateos, Buiza-Navarrete y Gentile, 2018). No obstante, el porcentaje de jugadores diarios era mucho más bajo $(22,7 \%)$, otro resultado comparable con el de los adolescentes españoles (Gonzálvez, Espada y Tejeiro, 2016).

Además, el $6,4 \%$ de la muestra total se clasificó como jugador problemático, un hallazgo similar a otros estudios con una prevalencia estimada entre 7,7\%-8,3\% de juego patológico entre adolescentes españoles (Buiza-Aguado et al., 2018; Lopez-Fernandez, Honrubia-Serrano, Baguley y Griffiths, 2014), y a la estimación de 5,1\% de riesgo de IGD en 
una muestra representativa de adolescentes europeos (Müller et al., 2015).

En cuanto al género, los chicos jugaban más a videojuegos y mostraban mayor juego problemático que las chicas, en sintonía con otros estudios (Mihara y Higuchi, 2017; Paulus et al., 2018). Las preferencias en géneros de videojuegos también se vieron afectadas por el género: las chicas preferían videojuegos de simulación social y lógica/habilidad mientras que los chicos preferían videojuegos competitivos y agresivos (ej., juegos de acción-shooter, deportes, estrategia y lucha), resultados coherentes con estudios anteriores (ej., Lemmens y Hendriks, 2016; Rehbein et al., 2016; Scharkow et al., 2015). Las diferencias de género sobre la personalidad pueden parcialmente explicar estos hallazgos. Las chicas tienden a mostrar puntuaciones más altas en apertura a la experiencia, y los chicos niveles más bajos en amabilidad (ej., Ortet et al., 2012; Costa y McCrae, 1992), lo que puede llevar a las chicas a preferir videojuegos más intelectuales y a los chicos a optar por juegos más competitivos y violentos. Estas diferencias de género en las preferencias de tipos de videojuegos pueden ayudar a explicar por qué los chicos, que prefieren juegos que consumen más tiempo y requieren mayor implicación, se involucran más con el juego que las chicas (Rehbein et al., 2016; Scharkow et al., 2015).

En relación a la personalidad, nuestros resultados replicaron el papel de la baja responsabilidad observada en adultos (Gervasi et al., 2017; Şalvarlı y Griffiths, 2019) y en los pocos estudios realizados sobre adolescentes (García-Oliva y Piqueras, 2016; Vollmer et al., 2014; Wang et al., 2015). La otra área de la personalidad relevante en la adicción a los videojuegos es el neuroticismo (Gervasi et al., 2017; Şalvarlı y Griffiths, 2019). No obstante, y en coherencia con otros estudios realizados con jóvenes (García-Oliva y Piqueras, 2016; Wang et al., 2015), no hallamos efecto alguno de esta dimensión en el juego problemático en adolescentes. Conjuntamente, estos resultados sugieren que una afectividad negativa es menos importante para el juego problemático en la adolescencia que en etapas vitales posteriores.

Otros factores de la personalidad que también se han asociado con el juego problemático en adolescentes son la introversión (Garcia-Oliva y Piqueras, 2016; Vollmer et al., 2014) y la baja amabilidad (Vollmer et al., 2014). Aunque nuestros datos sostienen la relevancia de estas características de personalidad, sugieren un papel diferencial según el género: la introversión se relacionó con juego problemático en chicas, mientras que la baja amabilidad tuvo una asociación importante en chicos. La segunda asociación puede implicar una mayor relevancia de los motivos de competitividad y agresividad para chicos, resultando en una mayor perseverancia en el juego, a pesar de sus consecuencias negativas (Vollmer et al., 2014). El papel de la introversión en el juego problemático exclusivamente para chicas puede explicarse por las diferencias de género asociadas a las preferencias en tipos de videojuego. Las chicas prefieren juegos de simulación social y lógica+habilidad con menos componentes sociales y excitantes que otros géneros preferidos principalmente por chicos, como juegos de action-shooter y deportes. Jugar a estos juegos constituye una actividad de ocio solitaria, por lo que chicas con pocas habilidades sociales (Gentile et al., 2011; Kowert, Vogelgesang, Festl y Quandt, 2015) y con características de introversión (Garcia-Oliva y Piqueras, 2016; Vollmer et al., 2014) tendrían mayor riesgo de juego problemático. Aunque estos resultados son sugerentes, requieren ser replicados; implican que futuros estudios han de prestar mayor atención a diferencias dependientes del género en relación a variables de riesgo y de protección implicadas en conductas relacionadas con el juego.

Otro factor de riesgo para el IGD es la frecuencia de juego (Gentile, 2009; Mihara y Higuchi, 2017; Vollmer et al., 2014). En nuestro estudio, hubo una asociación entre la frecuencia de juego y el juego problemático, pero con una magnitud entre baja y moderada. Este tamaño del efecto puede sugerir que los efectos perjudiciales de mayor uso de videojuegos afectarían a algunos adolescentes, pero no a otros. De esta manera, también encontramos que la cantidad de tiempo dedicada a jugar a videojuegos impactaría más negativamente sobre adolescentes menos responsables y más impulsivos. Encontramos efectos de esta interacción para chicos y chicas, que permanecieron robustos cuando controlamos por otras variables de confusión en la interacción (Keller, 2014). Se han descrito efectos de moderación similares en conductas relacionadas con el juego. Así, se ha mostrado que la exposición a videojuegos violentos principalmente afecta de manera negativa a aquellas personas con personalidad predispuesta a la agresión (ej., Anderson y Dill, 2000; Markey y Markey, 2010). Por otra parte, se han observado efectos similares en otras conductas adictivas, como en el uso de alcohol, donde factores ambientales de riesgo (ej., baja supervisión parental y alta disponibilidad de alcohol) parecen tener efectos perjudiciales sobre la conducta de uso de alcohol, principalmente en jóvenes desinhibidos (Pedersen y McCarthy, 2008). Conjuntamente, dichos hallazgos reflejarían procesos de interacción persona-ambiente (Caspi y Roberts, 2001), que sugieren que la baja responsabilidad y rasgos de impulsividad agravarían los efectos perjudiciales de los factores ambientales de riesgo sobre conductas adictivas.

Respecto a las preferencias en géneros de videojuegos, nuestro estudio encontró un papel pequeño, pero significativo de la personalidad. La apertura a la experiencia se asoció a juegos de RPG, lógica+habilidad, aventura, lucha, y simulación social. Los juegos de RPG, aventura, y lucha implican elementos fantásticos e irreales, por lo que jóvenes con una imaginación fértil pueden sentirse más atraídos hacia ellos (Braun et al., 2016). Las personas con mayor apertura a la experiencia, en particular las chicas, también preferirían géneros de juego menos convencionales y que implican mayor reto mental, como juegos de lógica+habilidad y simulación social, mientras que los adolescentes con baja apertura a la experiencia preferirían juegos convencionales y realistas, como juegos de deportes y acción-shooter. La extraversión juega un papel significativo en la elección de los juegos. Los géneros de acción-shooter y deportes se asociaron con la extraversión, probablemente porque dichos 
juegos contienen mayor componente social (ej., funciones de multijugador online) y tienden a ser más excitantes y estimulantes (Braun et al., 2016; Chory y Goodboy, 2011). Por el contrario, los adolescentes introvertidos tienden a preferir juegos de RPG, aventura, y simulación social. Estos datos sugieren que los jugadores introvertidos preferirían juegos solitarios, algunos para abordar sus necesidades interpersonales al simular interacciones sociales en un mundo virtual (Kowert et al., 2015). Los jugadores con baja amabilidad tienden a elegir juegos de lucha y estrategia (principalmente juegos MOBA), quizás debido a sus tendencias competitivas y a la gratificación violenta al enfrentarse en un juego (Chory y Goodboy, 2011; Greitemeyer y Sagioglou, 2017). La responsabilidad también fue relevante en cuanto a las preferencias de juegos, con altas puntuaciones asociadas a la elección de juegos "positivos" y "responsables", como juegos de entrenamiento de habilidades cognitivas y psicomotoras. Por el contrario, la baja responsabilidad fue predictora de preferencias en juegos de RPG y lucha, géneros que suelen implicar mucho tiempo de juego (Rehbein et al., 2016) y que se han asociado con un mayor riesgo para el IGD (Lemmens y Hendrix, 2016). Por último, el neuroticismo se asoció con juegos de construcción (ej., Minecraft), y se ha relacionado con síntomas del trastorno obsesivo-compulsivo (ej., Samuels et al., 2000), lo que podría explicar por qué los jugadores altos en neuroticismo prefieren juegos de actividades repetitivas.

Este estudio presenta algunas limitaciones. Primero, el muestreo fue por conveniencia, dado que la elección de institutos no fue aleatoria. Segundo, usamos cuestionarios para recopilar los datos, por lo que podrían estar afectados por sesgos, como el de deseabilidad social. Tercero, la asignación de nombres concretos a géneros de juego específicos fue, en cierta medida, artificial, porque los juegos suelen incluir una mezcla de características de diferentes géneros. Además, hubo una restricción importante de rango en el reporte de géneros de videojuegos (los participantes podían mencionar hasta cinco juegos). Dicha restricción de rango, junto con la relativa heterogeneidad de los juegos incluidos en cada género, podría explicar, en parte, los tamaños del efecto pequeños hallados en la asociación de género-personalidad. Cuarto, la categoría de «juego problemático» debería interpretarse más bien como un índice de uso problemático de videojuegos en vez de un diagnóstico clínico del IGD. Además, el desarrollo de la escala original (Gentile, 2009) data de antes de que se incluyera el IGD en el DSM-5. Por tanto, la prevalencia de nuestro estudio podría subestimarse debido al hecho de que el DSM- 5 establece 5 criterios para diagnosticar el IGD, en lugar de los 6 usados en la escala de este estudio. También, el punto de corte podría variar debido a diferencias culturales y a perfiles de jugadores (Bernaldo-de-Quirós, Labrador-Méndez, Sánchez-Iglesias y Labrador, 2020). Por último, debido al diseño transversal del estudio, no podemos realizar inferencias causales. Los rasgos de la personalidad pueden predisponer a conductas de juego, pero se ha demostrado que los videojuegos también pueden impactar sobre determinadas características de la personalidad (ej., Greitemeyer y Sagioglou, 2017). Más estudios deberán examinar las relaciones longitudinales entre personalidad y conductas de juego para establecer la dirección que subyace a las asociaciones entre estas variables estudiadas. A pesar de dichas limitaciones, este estudio presenta algunas fortalezas notables. Aborda un tema relativamente inexplorado: el papel del género y la personalidad sobre el uso y abuso de los videojuegos durante la adolescencia. Con este fin, administramos unos instrumentos con cualidades psicométricas consolidadas a una muestra relativamente amplia de preadolescentes y adolescentes, y seguimos un abordaje novedoso al examinar el papel moderador del género y la personalidad en el juego problemático.

Este estudio destaca la importancia del género y la personalidad a la hora de explicar conductas relacionadas con los videojuegos. Los chicos prefieren videojuegos más competitivos y agresivos, mientras que las chicas optan por juegos no violentos y ocasionales. Parece ser que algunas características específicas de personalidad afectan de manera diferente al juego problemático en chicos y chicas: hubo tasas más elevadas de juego problemático en chicas introvertidas con baja responsabilidad y en chicos poco amables con baja responsabilidad. La personalidad moderó el impacto negativo de la frecuencia de juego sobre el juego problemático: el uso regular de videojuegos se relacionó con el juego problemático principalmente en adolescentes con baja responsabilidad. Por último, diferentes perfiles de personalidad guían parcialmente la elección de videojuegos específicos. Estos hallazgos pueden ayudarnos a entender mejor el campo de los videojuegos en la adolescencia, y podrían ser útiles para diseñar de manera personalizada programas de tratamiento y estrategias de prevención ante el juego problemático basados en el género y las características de personalidad; de manera similar a los programas de prevención e intervención basados en la personalidad desarrollados para otras conductas relacionadas con las adicciones (Conrod, 2016).

\section{Reconocimientos}

Este estudio recibió financiación del Banco de Instrumentos de Cibersam; del Ministerio de Economía y Empresa de España (MINECO/FEDER) PSI2015-67766-R, del Ministerio de Ciencia, Innovación y Universidades RTI2018-099800-B-I00, de la Generalitat Valenciana GV/2016/158; y de la Universitat Jaume I UJI-A2017-18, UJI-B2017-74 y E-2018-16.

\section{Conflicto de intereses}

Los autores declaran la ausencia de conflicto de intereses.

\section{Referencias}

American Psychiatric Association (2013). Diagnostic and statistical manual of mental disorders (5th ed.). Arlington, 
VA: American Psychiatric Publishing. doi:10.1176/appi. books.9780890425596.

Anderson, C. A. y Dill, K. E. (2000). Video games and aggressive thoughts, feelings, and behavior in the laboratory and in life. Journal of Personality and Social Psychology, 78, 772-790. doi:10.1037/0022-3514.78.4.772.

Andreassen, C. S., Griffiths, M. D., Gjertsen, S. R., Krossbakken, E., Kvam, S. y Pallesen, S. (2013). The relationships between behavioral addictions and the five-factor model of personality. Journal of Behavioral Addictions, 2, 90-99. doi:10.1556/jba.2.2013.003.

Asociación Española de Videojuegos (2018). The Yearbook of Videogames 2018. Recuperado de: http://www.aevi.org. es/web/wp-content/uploads/2019/05/AEVI_Anuario_2018.pdf.

Bernaldo-de-Quirós, M., Labrador-Méndez, M., Sánchez-Iglesias, I. y Labrador, F.J. (2020). Measurement instruments of online gaming disorder in adolescents and young people according to DSM- 5 criteria: A systematic review. Adicciones, 32, 291-302. doi:10.20882/ adicciones. 1277 .

Bobes, B. M., Flórez, G., Seijo, P. y Bobes, G. J. (2019). Does ICD-11 improve the epidemiological and nosological purposes of mental, behavioral and developmental disorders? Adicciones, 31, 183-188. doi:10.20882/adicciones.1368.

Braun, B., Stopfer, J., Müller, K., Beutel, E. y Egloff, B. (2016). Personality and video gaming: Comparing regular gamers, non-gamers, and gaming addicts and differentiating between game genres. Computers in Human Behavior, 55, 406-412. doi:10.1016/j.chb.2015.09.041.

Buiza-Aguado, C., Alonso-Canovas, A., Conde-Mateos, C., Buiza-Navarrete, J. J. y Gentile, D. (2018). Problematic video gaming in a young Spanish population: Association with psychosocial health. Cyberpsychology, Behavior, and Social Networking, 21, 388-394. doi:10.1089/cyber.2017.0599.

Caspi, A. y Roberts, B. W. (2001). Personality development across the life course: The argument for change and continuity. Psychological Inquiry, 12, 49-66. doi:10.1207/ s15327965pli1202_01.

Charlton, J. P. y Danforth, I. D. (2007). Distinguishing addiction and high engagement in the context of online game playing. Computers in Human Behavior, 23, 1531- 1548. doi:10.1016/j.chb.2005.07.002.

Chory, R. y Goodboy, A. K. (2011). Is basic personality related to violent and non-violent video game play and preferences? Cyberpsychology, Behavior and Social Networking, 14, 191-198. doi:10.1089/cyber.2010.0076.

Conrod, P. J. (2016). Personality-targeted interventions for substance use and misuse. Current Addiction Reports, 3, 426-436. doi:10.1007/s40429-016-0127-6.

Costa, P. T. y McCrae, R. R. (1992). Revised NEO personality inventory (NEO-PI-R) and NEO five-factor inventory (NEO- FFI) professional manual. Odessa, FL: Psychological Assessment Resources.
Entertainment Software Association (2018). Essential facts about the computer and videogame industry. Recuperado de http:/ /www.theesa.com/wp-content/ uploads/2018/05/ EF2018_FINAL.pdf.

García-Oliva, C. y Piqueras, J. A. (2016). Experiential avoidance and technological addictions in adolescents. Journal of Behavioral Addictions, 5, 293-303. doi:10.1556/2006.5.2016.041.

Gentile, D. (2009). Pathological video-game use among youth ages 8 to 18: A national study. Psychological Science, 20, 594-602. doi:10.1111/j.1467-9280.2009.02340.x.

Gentile, D. A., Bailey, K., Bavelier, D., Brockmyer, J. F., Cash, H., Coyne, S. M.,... Young, K. (2017). Internet Gaming Disorder in children and adolescents. Pediatrics, 140, 8185. doi:10.1542/peds.2016-1758h.

Gentile, D. A., Choo, H., Liau, A., Sim, T., Li, D., Fung, D., ... Khoo, A. (2011). Pathological video game use among youths: A two-year longitudinal study. Pediatrics, 127, 319329. doi:10.1542/peds.2010-1353.

Gervasi, A. M., La Marca, L., Costanzo, A., Pace, U., Guglielmucci, F. y Schimmenti, A. (2017). Personality and Internet gaming disorder: A systematic review of recent literature. Current Addiction Reports, 4, 293-307. doi:10.1007/ s40429-017-0159-6.

González-Bueso, V., Santamaría, J., Fernández, D., Merino, L., Montero, E. y Ribas, J. (2018). Association between internet gaming disorder or pathological video-game use and comorbid psychopathology: A comprehensive review. International Journal of Environmental Research and Public Health, 15, 668-687. doi:10.3390/ijerph15040668.

Gonzálvez, M. T., Espada, J. P. y Tejeiro, R. (2017). Problem video game playing is related to emotional distress in adolescents. Adicciones, 29, 180-185. doi:10.20882/adicciones.745.

Greitemeyer, T. y Sagioglou, C. (2017). The longitudinal relationship between everyday sadism and the amount of violent video game play. Personality and Individual Differences, 104, 238-242. doi:10.1016/j.paid.2016.08.021.

Griffiths, M. D. (2005). A 'components' model of addiction within a biopsychosocial framework. Journal of Substance Use, 10, 191-197. doi:10.1080/14659890500114359.

Ibáñez, M. I., Moya, J., Villa, H., Mezquita, L., Ruipérez, M. Á. y Ortet, G. (2010). Basic personality dimensions and alcohol consumption in young adults. Personality and Individual Differences, 48, 171-176. doi:10.1016/j. paid.2009.09.017.

John, O. P., Naumann, L. P. y Soto, C. J. (2008). Paradigm shift to the integrative big five trait taxonomy. Handbook of personality: Theory and research, 3, 114-158.

Keller, M. C. (2014). Genex environment interaction studies have not properly controlled for potential confounders: the problem and the (simple) solution. Biological Psychiatry, 75, 18-24. doi:10.1016/j.biopsych.2013.09.006.

Kowert, R., Vogelgesang, J., Festl, R. y Quandt, T. (2015). Psychosocial causes and consequences of online video game play. Computers in Human Behavior, 45, 51-58. doi:10.1016/j.chb.2014.11.074. 
Lemmens, J. S. y Hendriks, S. J. F. (2016). Addictive online games: Examining the relationship between game genres and internet gaming disorder. Cyberpsychology, Behavior, and Social Networking, 19, 270-276. doi:10.1089/ cyber.2015.0415.

Lemmens, J. S., Valkenburg, P. M. y Peter, J. (2011). Psychosocial causes and consequences of pathological gaming. Computers in Human Behavior, 27, 144-152. doi:10.1016/j. chb.2010.07.015.

Lopez-Fernandez, O., Honrubia-Serrano, M. L., Baguley, T. y Griffiths, M. D. (2014). Pathological video game playing in Spanish and British adolescents: Towards the exploration of internet gaming disorder symptomatology. Computers in Human Behavior, 41, 304-312. doi:10.1016/j. chb.2014.10.011.

Markey, P. M. y Markey, C. N. (2010). Vulnerability to violent video games: A review and integration of personality research. Review of General Psychology, 14, 82-91. doi:10.1037/ a 0019000 .

McCabe, C., Kim, D. y King, K. (2018). Improving present practices in the visual display of interactions. Advances in Methods and Practices in Psychological Science, 1, 147-165. doi: $10.1177 / 2515245917746792$.

McCrae, R. R. y Costa, P. T. (2008). Empirical and theoretical status of the five-factor model of personality traits. En G. J. Boyle, G. Matthews, y D. H. Sakloske (eds.). Personality theory and assessment. Personality theories and models, Vol. 1, (pp. 273-294). London: Sage. doi:10.4135/9781849200462.n13.

Mezquita, L., Ibáñez, M. I., Villa, H., Fañanás, L., Moya-Higueras, J. y Ortet, G. (2015). Five-factor model and internalizing and externalizing syndromes: A 5-year prospective study. Personality and Individual Differences, 79, 98-103. doi:10.1016/j.paid.2015.02.002.

Mihara, S. y Higuchi, S. (2017). Cross-sectional and longitudinal epidemiological studies of internet gaming disorder: A systematic review of the literature. Psychiatry and Clinical Neurosciences, 71, 425-444. doi:10.1111/ pcn.12532.

Montero, I. y León, O. G. (2005). Sistema de clasificación del método en los informes de investigación en Psicología. International Journal of Clinical and Health Psychology, 5, 115-127.

Moya-Higueras, J., Cuevas, A., Marques-Feixa, L., Mezquita, L., Mayoral, M., Fañanás, L.,... Ibáñez, M. I. (2020). Recent stressful life events (SLE) and adolescent mental health: Initial validation of the LEIA, a new checklist for SLE assessment according to their severity, interpersonal, and dependent nature. Assessment, 27, 1777-1795. doi:10.1177/1073191118817648.

Müller, K. W., Janikian, M., Dreier, M., Wölfling, K., Beutel, M. E., Tzavara, C., Richardson, C. y Tsitsika, A. (2015). Regular gaming behavior and internet gaming disorder in European adolescents: Results from a cross-national representative survey of prevalence, predictors, and psychopathological correlates. European Child $\mathcal{E}$ Adolescent Psychiatry, 24, 565-574. doi:10.1007/s00787-014-0611-2.
Organización Mundial de la Salud (OMS) (2017, July 14). ICD-11 Beta Draft. Mental, behavioural or neurodevelopmental disorders. Recuperado de https://icd.who.int/dev11/l-m/en.

Ortet, G., Ibáñez, M. I., Moya, J., Villa, H., Viruela, A. y Mezquita, L. (2012). Assessing the five factors of personality in adolescents: The junior version of the Spanish NEO-PI-R. Assessment, 19, 114-130. doi:10.1177/1073191111410166.

Ozer, D. J. y Benet-Martinez, V. (2006). Personality and the prediction of consequential outcomes. Annual Review of Psychology, 57, 401-421. doi:10.1146/annurev. psych.57.102904.190127.

Paulus, F. W., Ohmann, S., Von Gontard, A. y Popow, C. (2018). Internet gaming disorder in children and adolescents: A systematic review. Developmental Medicine $\mathcal{E}$ Child Neurology, 60, 645-659. doi:10.1111/dmcn.13754.

Pedersen, S. L. y McCarthy, D. M. (2008). Person-environment transactions in youth drinking and driving. Psychology of Addictive Behaviors, 22, 340-348. doi:10.1037/0893164x.22.3.340.

Rehbein, F., Staudt, A., Hanslmaier, M. y Kliem, S. (2016). Video game playing in the general adult population of Germany: Can higher gaming time of males be explained by gender specific genre preferences? Computers in Human Behavior, 55, 729-735. doi:10.1016/j. chb.2015.10.016.

Şalvarlı, Ş. İ. y Griffiths, M. D. (2019). Internet Gaming Disorder and its associated personality traits: A systematic review using PRISMA guidelines. International Journal of Mental Health and Addiction, 1-23. doi:10.1007/s11469019-00081-6.

Samuels, J., Nestadt, G., Bienvenu, O. J., Costa, P. T., Riddle, M. A., Liang, K. Y.,... Cullen, B. A. (2000). Personality disorders and normal personality dimensions in obsessive-compulsive disorder. British Journal of Psychiatry, 177, 457-462. doi:10.1192/bjp.177.5.457.

Scharkow, M., Festl, R., Vogelgesang, J. y Quandt, T. (2015). Beyond the "core-gamer": Genre preferences and gratifications in computer games. Computers in Human Behavior, 44, 293-298. doi:10.1016/j.chb.2014.11.020.

Vollmer, C., Randler, C., Horzum, M. B. y Ayas, T. (2014). Computer game addiction in adolescents and its relationship to chronotype and personality. SAGE Open, 4, 1-9. doi: $10.1177 / 2158244013518054$.

Walker, J. O., López, F.J. y Mezquita, L. (2018, September). An abridge form of the Junior Spanish version of the NEO PI-R (JS NEO-A60). Poster submitted at the VII Jornada de la AIIDI, Palma de Mallorca, España.

Wang, C. W., Ho, R. T. H., Chan, C. L.W. y Tse, S. (2015). Exploring personality characteristics of Chinese adolescents with internet-related addictive behaviors: Trait differences for gaming addiction and social networking addiction. Addictive Behaviors, 42, 32-35. doi:10.1016/j. addbeh.2014.10.039.

Watkins, M. W. (2006). Determining parallel analysis criteria. Journal of Modern Applied Statistical Methods, 5, 344-346. doi:10.22237/jmasm/1162354020. 